\title{
Synthesis and Characterization of a Novel Tetrapod-Like ZnO Nanostructure
}

\author{
H. Liu,* L. F. Allard** and J. Liu***
}

*Center for Nanoscience, University of Missouri-St. Louis, MO 63121

**Materials Science and Technology Div., Oak Ridge National Laboratory, Oak Ridge, TN 37831

***Center for Nanoscience, Department of Physics \& Astronomy, Department of Chemistry \& Biochemistry, University of Missouri-St. Louis, MO 63121 (liuj@,umsl.edu)

Nanostructures, as basic building blocks for integrated systems, have attracted intense interest in a variety of fields of research. There are many reports on observing new types of $\mathrm{ZnO}$ nanostructures; however, controlled large-scale synthesis of these reported nanostructures is still a challenge. Our group has been developing synthesis protocols in order to reliably fabricate large amounts of welldefined nanostructures for applications in catalysis, energy harvesting and storage, and nanomedicine. We report here our recent success in synthesizing, on a gram scale, a tetrapod-like $\mathrm{ZnO}$ nanostructure by a modified vapor deposition method.

The tetrapod-like $\mathrm{ZnO}$ nanostructure was synthesized in a high-temperature furnace. A field emission SEM was used to characterize the general morphology and the uniformity of the fabricated $\mathrm{ZnO}$ nanostructures. The atomic-level surface information of the tetrapod-like nanostructures was obtained using a JEOL 2200FS (scanning) transmission electron microscope (STEM/TEM) equipped with a CEOS Co. aberration corrector for the probe-forming lenses, which provides a nominal resolution of about $0.07 \mathrm{~nm}$ [1].

Figure 1a shows a representative low-magnification SEM image of the synthesized $\mathrm{ZnO}$ nanostructures with sizes ranging from 3 to 6 micrometers. Many SEM images obtained from different batches showed similar nanostructures, suggesting that large amounts of the unique $\mathrm{ZnO}$ nanostructure have been repeatedly synthesized. Figure $1 \mathrm{~b}$ and 1c show high-magnification SEM images of the individual $\mathrm{ZnO}$ nanostructure oriented along different directions, clearly revealing the presence of nanosheets which connects the long and curved nanowires. Analysis of many such SEM images revealed that the majority of the synthesized nanostructures contain three triangular nanosheets and four very long nanoarms with diameters varying from 50 to $80 \mathrm{~nm}$. Since these four long nanoarms seem to be connected at the center of the nanostructure, similar to those of previously reported tetrapod structures [2], we call the unique nanostructure that we have synthesized a "tetrapod-like" structure. The growth of the triangular nanosheets connects the different legs of our tetrapod-like nanostructures. High-angle annular dark-field (HAADF) images (Fig. 2) at subÅngström resolution revealed that the nanosheets are composed of $\{10-10\}$ surfaces bounded by the atomically flat $\{0001\}$ and the faceted $\{20-21\}$ surfaces. Analyses of many HAADF images (similar to those shown in Fig. 2) indicate that the semi-polar $\{20-21\}$ surfaces were not stable; they decomposed into non-polar $\{10-10\}$ and polar $\{10-11\}$ facets. The growth mechanisms and the structural model of the synthesized tetrapod-like nanostructure will be discussed [3].

\section{References}

[1] D.A. Blom, L.F. Allard, S. Mishina and M.A. O'Keefe, Micros Microanal 12 (2006) 483.

[2] M.C. Newton, P.A. Warburton, Mater. Today 10 (2007) 50.

[3] This research was sponsored by the University of Missouri-St. Louis. Microscopy work at Oak Ridge National Laboratory's High Temperature Materials Laboratory was sponsored by the U.S. 
Department of Energy, Office of Energy Efficiency and Renewable Energy, Vehicle Technologies Program.
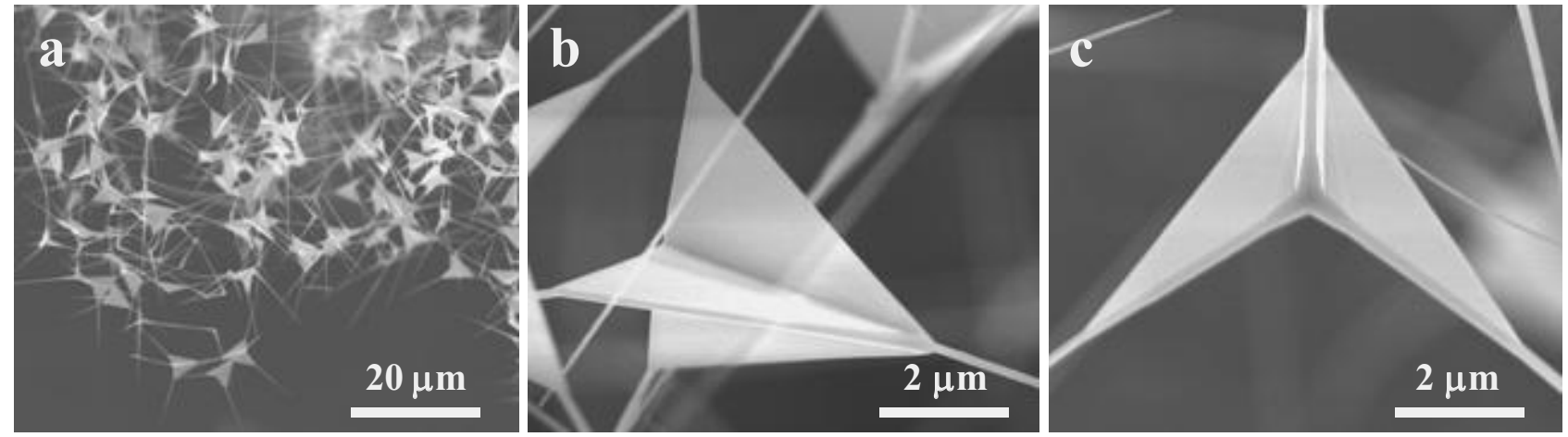

FIG.1. (a) low magnification SEM image of the synthesized $\mathrm{ZnO}$ nanostructure. The high magnification SEM images (b) and (c) show, along different electron beam projections, the triangular shape of the nanosheets and their spatial relationship with each other. Analyses of many such SEM images revealed that the unique $\mathrm{ZnO}$ nanostructure consists of three triangular nanosheets and four very long nanowires, all connected at the center of the nanostructure.
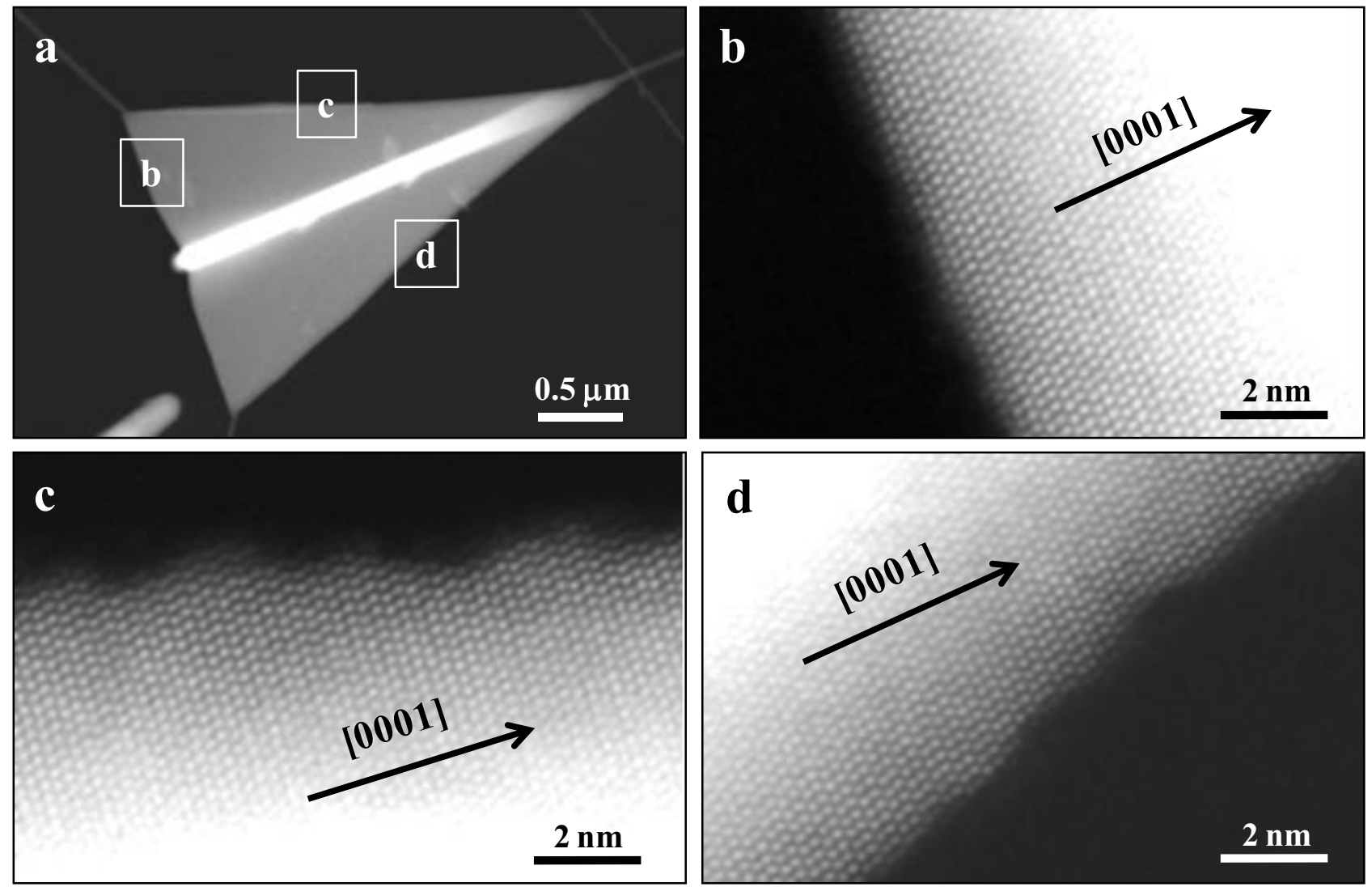

FIG.2. Low magnification HAADF image (a) and atomic-resolution HAADF images (b, c and d) of the nanosheets of a tetrapod-like nanostructure. The nanosheets are composed of $\{10-10\}$ surfaces bounded by the atomically flat $\{0001\}$ and faceted $\{20-21\}$ side surfaces. 